\title{
Network Embedding Based Recommendation Method in Social Networks
}

\author{
Yufei Wen \\ Lei Guo* \\ wenyufei92@sina.com \\ leiguo.cs@gmail.com \\ Shandong Normal University \\ Jinan, China
}

\author{
Zhumin Chen \\ Jun Ma \\ chenzhumin@sdu.edu.cn \\ majun@sdu.edu.cn \\ Shandong University \\ Jinan, China
}

\begin{abstract}
With the advent of online social networks, the use of information hidden in social networks for recommendation has been extensively studied. Unlike previous work regarded social influence as regularization terms, we take advantage of network embedding techniques and propose an embedding based recommendation method. Specifically, we first pre-train a network embedding model on the users' social network to map each user into a low dimensional space, and then incorporate them into a matrix factorization model, which combines both latent and pre-learned features for recommendation. The experimental results on two real-world datasets indicate that our proposed model is more effective and can reach better performance than other related methods.
\end{abstract}

\section{CCS CONCEPTS}

- Information systems $\rightarrow$ Data mining; Collaborative filtering;

\section{KEYWORDS}

Social Recommendation, Network Embedding, Matrix Factorization

\section{ACM Reference Format:}

Yufei Wen, Lei Guo, Zhumin Chen, and Jun Ma. 2018. Network Embedding Based Recommendation Method in Social Networks. In $W W W$ '18 Companion: The 2018 Web Conference Companion, April 23-27, 2018, Lyon, France. ACM, New York, NY, USA, 2 pages. https://doi.org/10.1145/3184558.3186904

\section{INTRODUCTION}

In social networks, users are more likely to seek suggestions from their friends. Social relationships provide an independent source of information about users beyond rating information. Therefore, how to utilize social information to assist recommendation has been widely studied in recent years [2, 3, 5]. For example, Mohsen et al.[2] incorporated

\footnotetext{
* Corresponding author
}

This paper is published under the Creative Commons Attribution 4.0 International (CC BY 4.0) license. Authors reserve their rights to disseminate the work on their personal and corporate Web sites with the appropriate attribution.

$W W W$ '18 Companion, April 23-27, 2018, Lyon, France

(C) 2018 IW3C2 (International World Wide Web Conference Committee), published under Creative Commons CC BY 4.0 License.

ACM ISBN 978-1-4503-5640-4/18/04.

https://doi.org/10.1145/3184558.3186904 the mechanism of trust propagation into Matrix Factorization (MF) to predict the behavior of users. However, most of these existing works mainly regard social influence as regularization terms, and the deeper structural information of social networks has not been fully explored.

Motivated by the success of network embedding techniques, we first pre-train the network embedding model node2vec[1] to learn high-level network representations from social relations, and then incorporate them into the MF based model. By combining the latent and pre-learned network features together, our method not only can make use of the social network information deeply, but also can take advantage of the collaborative filtering model for recommendation. Experimental results on two real-world datasets demonstrate the effectiveness of our proposed approach.

\section{RECOMMENDATION METHOD}

In this section, we first introduce the classic latent factor model, and then focus on how to combine the pre-trained network representations into MF to conduct social recommendation.

\subsection{Low-rank Matrix Factorization Model}

Let $\mathcal{U}=\left\{u_{1} \ldots u_{M}\right\}$ denote the user set, $\mathcal{I}=\left\{i_{1} \ldots i_{N}\right\}$ denote the item set, and $R=\left[R_{u, i}\right]_{M \times N}$ denote the user-item rating matrix, where $R_{u, i}$ represents the ratings of user $u$ on item $i$. A low-rank matrix factorization approach seeks to approximate the rating matrix $R$ by a multiplication of $k$-rank factors, and its objective function can be arrived as:

$$
\min _{U, I} \frac{1}{|D|} \sum_{(u, i) \in D} \mathcal{L}\left(R_{u, i}, \widehat{R}_{u, i}(U, I)\right)+\Omega(U, I)
$$

Where $D$ is the observed user-item rating pairs, $U$ and $I$ are the latent feature factors of users and items, with column vectors $U_{u} \in \mathbb{R}^{k}$ and $I_{i} \in \mathbb{R}^{k}$ representing user-specific and item-specific feature vectors, respectively. $\widehat{R}_{u, i}(U, I)=U_{u}^{T} I_{i}$ is the predicted score for the dyad $(u, i), \mathcal{L}(\cdot, \cdot)$ is the square loss function, and $\Omega(U, I)=\frac{\lambda_{U}}{2}\|U\|_{F}^{2}+\frac{\lambda_{I}}{2}\|I\|_{F}^{2}$ is the corresponding regularization term.

\subsection{Combined with Pre-learned Features}

As users in social networks often express their social interest by making different friends, a better understanding of these social networks is potentially helpful for recommendation. Let $\mathcal{G}$ present the social relationships among users, where an 
edge denotes there is a friend relationship between user $u$ and $v$. To mine the deep social structure from $\mathcal{G}$, we introduce the network embedding model node2vec[1] to learn the high-level user representations ${ }^{1}$, and let $X_{u} \in \mathbb{R}^{d}$ represent the learned feature vector of user $u$, which denotes how well a user is influenced by his friends in graph $\mathcal{G}$. By fusing these pre-trained features with the latent features from collaborative filtering model linearly, we can arrive at our embedding based recommendation method MFn2v:

$$
\begin{aligned}
\mathcal{L}(U, I, W)= & \min _{U, I, W} \frac{1}{2} \sum_{u=1}^{M} \sum_{i=1}^{N}\left(R_{u, i}-U_{u}^{T} I_{i}-W_{u}^{T} X_{u}\right)^{2} \\
& +\frac{\lambda_{U}}{2}\|U\|_{F}^{2}+\frac{\lambda_{I}}{2}\|I\|_{F}^{2}+\frac{\lambda_{W}}{2}\|W\|_{F}^{2}
\end{aligned}
$$

where $W_{u} \in \mathbb{R}^{d}$ is the weighted vector that indicates how much the pre-trained network features should contribute to user $u$.

We apply stochastic gradient descent method to find a local minimum of Eq. 2, and update the latent factors $U, I$ and $W$ by the following gradients:

$$
\begin{aligned}
& \frac{\partial \mathcal{L}(U, I, W)}{\partial U_{u}}=\sum_{i=1}^{N}\left(U_{u}^{T} I_{i}+W_{u}^{T} X_{u}-R_{u, i}\right) I_{i}+\lambda_{U} U_{u} \\
& \frac{\partial \mathcal{L}(U, I, W)}{\partial I_{i}}=\sum_{u=1}^{M}\left(U_{u}^{T} I_{i}+W_{u}^{T} X_{u}-R_{u, i}\right) U_{u}+\lambda_{I} I_{i} \\
& \frac{\partial \mathcal{L}(U, I, W)}{\partial W_{u}}=\sum_{i=1}^{N}\left(U_{u}^{T} I_{i}+W_{u}^{T} X_{u}-R_{u, i}\right) X_{u}+\lambda_{W} W_{u}
\end{aligned}
$$

\section{EVALUATION}

\subsection{Experimental Setup and Comparisons}

We utilize two real-world datasets (Ciao and Epinions) to evaluate our recommendation method, and for Ciao[5] the latent factor dimension $k$ is set as 15 and the regularization parameters are set as $\lambda_{U}=\lambda_{I}=0.6$ and $\lambda_{W}=0.001$. For Epinions[5], the parameters are set as: $k=15, \lambda_{U}=\lambda_{I}=0.6$, $\lambda_{W}=0.005$.

For both of these two datasets, $80 \%$ of randomly selected ratings are used for training, and RMSE and MAE[5] are utilized as the evaluation metrics. In this work, we compare our method with three related approaches: MF, LFL[4] and SocialMF[2].

Table 1: The Performance Comparison on Ciao and Epinion

\begin{tabular}{c|c|c|c|c|c}
\hline Dataset & Metrics & MF & LFL & SocialMF & MFn2v \\
\hline \multirow{2}{*}{ Ciao } & MAE & 0.782 & 0.760 & 0.755 & 0.746 \\
\cline { 2 - 6 } & RMSE & 1.006 & 1.002 & 0.990 & 0.974 \\
\hline \multirow{2}{*}{ Epinions } & MAE & 0.846 & 0.842 & 0.830 & 0.823 \\
\cline { 2 - 6 } & RMSE & 1.086 & 1.070 & 1.062 & 1.058 \\
\hline
\end{tabular}

${ }^{1}$ The parameter settings of our pre-trained node2vec model are: $d=$ $10 ; l=80 ; r=10 ; k=10 ; p=1 ; q=0.5$.
The experimental results are shown in Table 1, from which we can find: As MF only uses the rating information for recommendation, it does worse than other methods. The stateof-the-art social recommendation method SocialMF achieves a better performance than both MF and LFL, which demonstrates the social relationship is helpful to model users' preference. From this result, we can also find that our proposed method MFn2v can perform better than SocialMF, and reach the best performance in experiments, which indicates that fusing the pre-trained embedding with latent factors is helpful, and can effectively model both the users' personal and social interests.

\subsection{Convergence Analysis}

To explore the efficiency of our model, we further conduct experiments to compare the convergence of our MFn2v method with MF method on Ciao. To make them comparable, same learning rates are adopted. Fig. 1 shows the comparison results, from which we can observe both these two methods converge very fast (converge within 80 iterations). The convergence rate of $\mathrm{MFn} 2 \mathrm{v}$ is not slowed down by incorporating the social network representations, on the contrary it can make a better performance than MF method (Similar results can also be reached on the Epinions data).
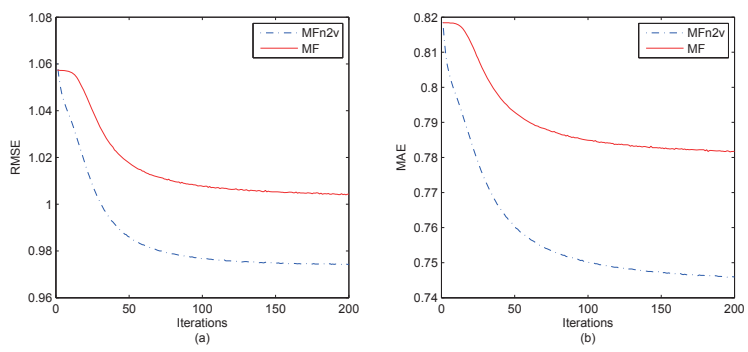

Figure 1: Convergence analysis on the Ciao data (a) RMSE (b) MAE.

\section{ACKNOWLEDGMENTS}

This work is supported by the Natural Science Foundation of China (Nos. 61602282, 61672324, 61672322), and the China Postdoctoral Science Foundation (No. 2016M602181).

\section{REFERENCES}

[1] A Grover and J Leskovec. 2016. node2vec: Scalable Feature Learning for Networks. In KDD. ACM, 855-864.

[2] Mohsen Jamali and Martin Ester. 2010. A matrix factorization technique with trust propagation for recommendation in social networks. In RecSys. ACM, 135-142.

[3] Jarana Manotumruksa, Craig Macdonald, and Iadh Ounis. 2016. Regularising Factorised Models for Venue Recommendation using Friends and their Comments. In CIKM. ACM, 1981-1984.

[4] Aditya Krishna Menon and Charles Elkan. 2011. A Log-Linear Model with Latent Features for Dyadic Prediction. In ICDM. IEEE, 364-373.

[5] Suhang Wang, Jiliang Tang, and Huan Liu. 2015. Toward dual roles of users in recommender systems. In CIKM. ACM, 16511660 . 